\title{
COMBINATIONS OF ENERGY SAVING MEASURES IN PNEUMATICS
}

\author{
Vladimir Boyko*, Jürgen Weber \\ Institute of Mechatronic Engineering, Technische Universität Dresden, Helmholtzstrasse 7a, 01069 Dresden \\ * Corresponding author: Tel.: +49 351 46334109; E-mail address: vladimir.boyko@tu-dresden.de
}

\begin{abstract}
Within a production machine, various electromechanical and pneumatic structures can be used for drive tasks. As these drive technologies can often replace each other, the energy efficiency, performance and TCO of pneumatic drives must be permanently enhanced to remain competitive. There is a large number of known measures for the reducing their energy consumption, e.g. minimizing of filling volumes, energy saving circuits etc. However, these measures are mainly considered separately and the possible overall energy saving effect resulting from their combination is rarely taken into account.

The main goal of this paper is therefore to explore the possible combinations of pneumatic energy saving measures and their cumulative saving effect. Due to the fact that some measures are mutually exclusive (e.g. a general pressure reduction in properly sized drive), each combination should be considered separately. To evaluate their efficiency plausibly, a comprehensive assessment is required that contains both the total cost of ownership analysis (TCO) and a mechanical properties assessment. The presented comprehensive approach of the task analysis can serve as example of how an optimal drive configuration for the specific task can be found, thus creating a basis for solving constructive challenges in designing of pneumatic automation systems.
\end{abstract}

Keywords: Pneumatics, Energy saving circuit, Efficiency, Exergy, TCO

\section{INTRODUCTION}

In the design of automation systems, engineers nowadays often have the choice of performing the drive technology pneumatically or electrically. For both drive solutions, a variety of predestined application fields results from their technological properties and the cost-specific situation. Due to the large number of ready-made control concepts for position, speed and force, high rigidity and accuracy of the drive system as well as high availability, electric drives are often preferred in the implementation of positioning tasks. In contrast, pneumatic drives have such advantages as simple realization of point-to-point movement, usage of multiple parallel drives (many actuators on a valve terminal) and a range of possible movement and handling solutions (linear movement, clamping, suction, gripping etc.). Also, much lower investment costs and realization of holding functions without steady energy consumption are clearly beneficial in comparison to the electromechanics.

The higher energy consumption of pneumatic drives is often criticized where both drive technologies compete for their implementation.
To keep up with the competition, manufacturers and users of pneumatic systems are required to increase the energy efficiency of pneumatics at all three levels (compressed air generation, conducting part and consuming actuator part). A reduction of the energy consumption of the drive also contributes to the achievement of climate protection goals, as they stated e.g. in the Europe 2020 initiative [1].

The following article deals exclusively with the energy efficiency at the actuator level for linear movement actuators. There is already a large variety of known energy saving measures, which make it possible to reduce the compressed air consumption of actuators significantly and thus the total operating costs of a pneumatically operated plant. These include primarily the operating pressure reduction of a system and the reducing its filling volumes by shortening the hoses and using micro-valves (e.g. [2]). On the other hand, it is conceivable to use various investigated energy saving circuits such as exhaust air storage [3], [4] or cutting off the supply air to use its expansion energy [5], [6]. 
However, the application of these measures and their impact on energy consumption were mainly studied individually. It would be therefore reasonable to analyse how energy saving measures can be combined in order to be used simultaneously and whether such a combination can possibly achieve larger cumulative savings effects compared to individual measures.

The study [7] mentions (without making a quantifiable statement) that a combination of measures can be expected to increase savings. According to the results of the study, the measures influence each other, so that an implementation must always be examined in the overall context. Individual measures can even be mutually exclusive (e.g. general pressure reduction and a needs-based design of drives). Because of the mutual influence, no statement of an increase in the overall efficiency of a plant on the basis of the values presented is possible. The percentage efficiency gains cannot be added easily to determine total energy savings and should be determined separately for each case.

The dissertation [8] demonstrates the principle feasibility of the combinatorial approach and shows the possible cumulative savings. However, the results obtained there are valid only for a horizontal movement task with a symmetrical constant loading.

The main goal of the present article is therefore to supplement and investigate the possible combinations of energy saving measures on various drive tasks typical for a pneumatic automation system.

In order to evaluate and contrast the combinations objectively, a complete costbenefit analysis is required. A statement on the basis of achievable savings alone would be incomplete. For this purpose, a method for the technical-economic evaluation of the combinations is also developed in the present work.

\section{COMBINATORIAL APPROACH}

\subsection{Combinations generation}

The energy saving measures to be investigated are based on the classification shown in [4] and [9]. For the generation, the measures and their combinations are divided into the following groups:
Table 1: Combination groups

\begin{tabular}{|c|c|c|}
\hline Group & Description & Code \\
\hline \multirow[t]{6}{*}{ G1 } & Separate circuits: & \\
\hline & Standard circuit & 0 \\
\hline & Short circuit & 1 \\
\hline & Expansion (cut-off) circuit & 2 \\
\hline & Exhaust air storage & 3 \\
\hline & Pressure-reduced stroke & 4 \\
\hline \multirow[t]{3}{*}{$\mathrm{G} 2$} & $\frac{\text { Combinations of separate circuits, }}{\text { e.g.: }}$ & \\
\hline & Short and expansion circuit & 12 \\
\hline & $\begin{array}{l}\text { Pressure-reduces backstroke with } \\
\text { short and expansion circuit }\end{array}$ & 124 \\
\hline \multirow[t]{2}{*}{ G3 } & $\begin{array}{l}\text { Combinations of groups G1 and } \\
\text { G2 with the measure "Filling } \\
\text { volumes reduction" (code no. 7), } \\
\text { e.g.: }\end{array}$ & \\
\hline & $\begin{array}{l}\text { Short circuit with reduced filling } \\
\text { volume }\end{array}$ & 17 \\
\hline \multirow[t]{2}{*}{ G4 } & $\begin{array}{l}\text { Combinations of groups G1 and } \\
\text { G2 with the measure "General } \\
\text { pressure reduction" (code no. 6), } \\
\text { e.g.: }\end{array}$ & \\
\hline & $\begin{array}{l}\text { Short circuit with reduced working } \\
\text { pressure }\end{array}$ & 16 \\
\hline \multirow[t]{4}{*}{ G5 } & Combinations of the group G3 & \\
\hline & with the measure "General & \\
\hline & $\begin{array}{l}\text { pressure reduction" (code no. 6), } \\
\text { e.g.: }\end{array}$ & \\
\hline & $\begin{array}{l}\text { Expansion circuit with exhaust air } \\
\text { storage, reduced filling volume } \\
\text { and reduced working pressure }\end{array}$ & 2376 \\
\hline
\end{tabular}

Only meter-out throttling circuits are considered, with the exception of exhaust air storage circuits (3), where the forward stroke is realized as a meter-in throttling. Furthermore, each measure/measure combination is examined in three possible versions:

- with $3 / 2$ way control valves (code "a")

- with $5 / 2$ way control valves (code " $b$ ")

- with $5 / 3$ way control valves (code "c")

Some examples of generated combinations are shown in Figure 1. 
$\mathrm{Ob}$

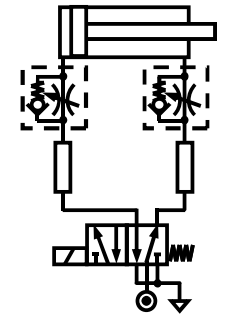

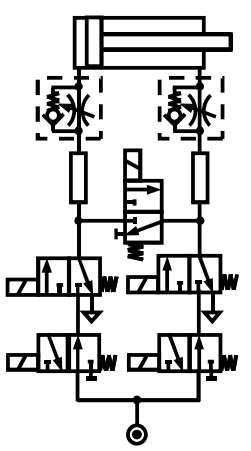

$12 \mathrm{a}$

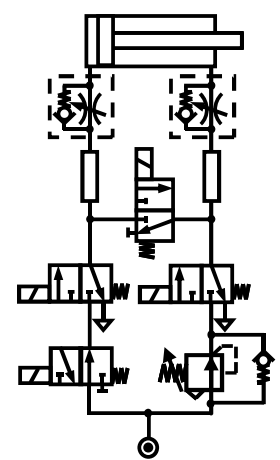

$124 \mathrm{a}$ $2376 \mathrm{c}$

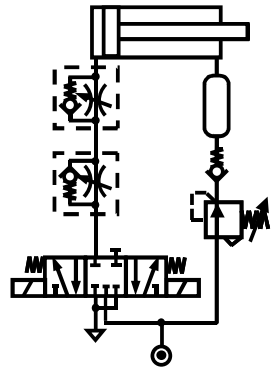

Figure 1: Examples of generated combinations: (0b) Standard circuit with 5/2 way control valve; (12a) Short and expansion circuit with 3/2 way control valves; (124a) Pressure-reduced backstroke with short and expansion circuit with 3/2 way control valves; (2376c) Expansion circuit with exhaust air storage, reduced filling volume and reduced working pressure with 5/3 way control valve.

In this way, 150 theoretically possible combinations were generated.

\subsection{Selection of use cases}

The generated combinations are to be tested on customary tasks in the drive technology. For this purpose, three common pneumatically operated automation systems are analysed in order to select typical tasks and to test the combinatorial method using their example. As a result of the analysis, the following tasks shown in Table 2 were chosen. The table also contains such operations conditions as the working pressure of the drive $p$ and its hose length $l$ between the control valve and the cylinder.

Table 2: Use cases

\begin{tabular}{ll}
\hline Drive task & Operation conditions \\
\hline 1) Feed drive: & $p=7$ bar $_{\text {abs. }}$ \\
movement and ejection of a & $l=1 \mathrm{~m}$ \\
workpiece & Cylinder dimensions: \\
Orientation: horizontal & $\varnothing 10$, stroke $20 \mathrm{~mm}$ \\
2) Force drive: & $p=7$ bar $_{\text {abs. }}$ \\
pressing force $\left(F_{\min }=180 \mathrm{~N}\right)$ & $l=1 \mathrm{~m}$ \\
at the end of the stroke & Cylinder dimensions: \\
Orientation: horizontal & $\varnothing 25$, stroke $50 \mathrm{~mm}$ \\
3) Feed drive: & $p=5$ bar abs. \\
constant mass $(m=10 \mathrm{~kg})$ & $l=1,2 \mathrm{~m}$ \\
movement on whole stroke & Cylinder dimensions: \\
and backstroke & $\varnothing 40$, stroke $300 \mathrm{~mm}$ \\
$\begin{array}{l}\text { Orientation: vertical } \\
\text { 4) Feed drive: }\end{array}$ & $p=5$ bar abs. \\
constant mass $(m=9,2 \mathrm{~kg})$ & $l=5$ cm \\
movement on whole stroke & Cylinder dimensions: \\
and backstroke & $\varnothing 32$, stroke $100 \mathrm{~mm}$ \\
Orientation: horizontal & \\
\hline
\end{tabular}

With this selection, the large range of typical pneumatic drive applications should be covered. The drive size for each task was selected on the basis of the median value of all actuators used for the specific task on the analysed automation systems. The operation conditions are also based on the original manufacturer specifications.

\subsection{Cost-benefit analysis}

In the next step, each generated combination is examined for its suitability for the specific task and graded on a scale of 0 to 3 :

- 3 - circuit usage possible without hesitation

- 2-circuit usage partially limited (one limitation, e.g. possible in one stroke direction only)

- 1 - circuit usage very limited (two or more limitations, e.g. a pressure reserve required and possible only in one stroke direction)

- 0 - circuit not functional or redundant

For instance, a short circuit (1) could not be applied for the force drive task 2 because of its force loss at the stroke end. Therefore, the variant (1) and all combinations with it are not functional and obtain the grade " 0 ". Whereas, the short circuit application for the feed drive task 1 is possible, but only for one direction (forward). The grade for the variant (1) in this case is " 2 ".

After the preliminary grading, there follows the subsequent calculation of each combination that has been graded at least with " 2 ". The combinations are evaluated from a technicalmechanical and economic point of view. As a final result, the technical benefit value (as a dimensionless score between 0 and 1) and the total cost of ownership balance (as a TCO-value 
per year) should be determined for each combination.

\section{Technical benefit}

For a technical-mechanical balancing, the following relevant properties of each circuit must be calculated:

- Exergy consumption of a drive $E x[\mathrm{~J} /$ cycle $]$

- Achievable stroke times $t_{\text {for }}$ and $t_{\text {back }}[\mathrm{s}]$

- Payback time $t_{\mathrm{p}}$

- For task 2: pressing force safety factor at the stroke end $S_{\text {force }}[-]$

- For tasks 3 and 4: maximum acceleration at the stroke end $a_{\max }\left[\mathrm{m} / \mathrm{s}^{2}\right]$

The exergy consumption of a drive is calculated accordingly to [8]:

$$
\begin{aligned}
& E x=\int\left[\dot{m}_{1} \cdot c_{\mathrm{p}} \cdot\left(T_{1}-T_{0}\right)+\right. \\
& \left.\dot{m}_{1} \cdot T_{0} \cdot\left(R_{\mathrm{s}} \cdot \ln \left(\frac{p_{1}}{p_{0}}\right)-c_{\mathrm{p}} \cdot \ln \left(\frac{T_{1}}{T_{0}}\right)\right)\right] d t
\end{aligned}
$$

The ambient temperature $T_{0}$ and atmospheric pressure $p_{0}$ represent the calculation reference (environment). The values mass flow $\dot{m}_{1}$, temperature $T_{1}$ and pressure $p_{1}$ are defined at the circuit inlet before the way control valve(s). Thermodynamic properties of air include specific heat capacity at constant pressure $c_{\mathrm{p}}$ and specific gas constant $R_{\mathrm{s}}$.

The force safety factor $S_{\text {force }}$ is defined as the relationship between the current and the required force. The payback time expresses the reasonableness and suitability of a retrofit with the concrete combination. It is simplified calculated as the ratio between the additional acquisition costs and the energy cost saving per year compared to the reference standard circuit (0).

In the course of the benefit analysis, it is checked to what extent each combination fulfils the predefined mechanical objectives. For this purpose, a utility function is set up for each named technical-mechanical variable, which makes it possible to determine a dimensionless fulfilment degree of a requirement $E$ corresponding to [10]. The designed utility functions are shown in Figure 2 using the example of the force drive task 2.

The individual requirements for the task are derived from the manufacturer information.
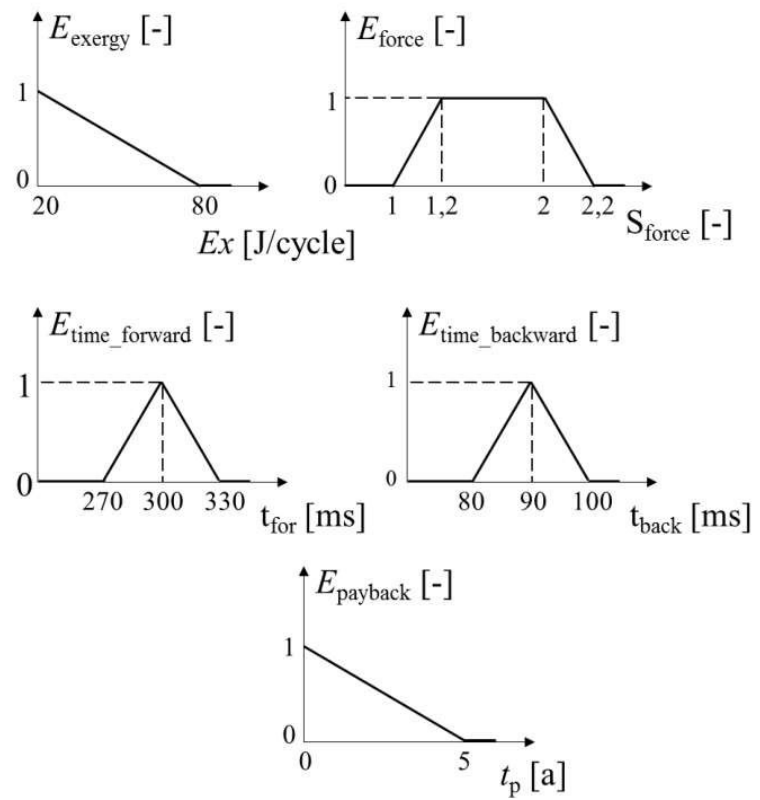

Figure 2: Utility functions of the task 2

To determine the total technical benefit value of a combination $N_{\text {total, }}$ it is necessary to set the weights of each goal $w_{\mathrm{i}}$, which are defined in the present case as follows:

Table 3: Weight factors for task 2

\begin{tabular}{ll}
\hline Value & Weight factor $w_{\mathrm{i}}, \%$ \\
\hline Exergy consumption $E x$ & 25 \\
Stroke time forwards $t_{\text {for }}$ & 10 \\
Stroke time backwards $t_{\text {back }}$ & 10 \\
Payback time $t_{\mathrm{p}}$ & 25 \\
Pressing force $F_{\text {press }}$ & 30 \\
\hline
\end{tabular}

The stroke times have the smallest weighting due to the fact that the drive settings (e.g. throttle opening, pressure level) were chosen so that the mechanical properties could be kept according to the specification. For instance, by even more reduced operating pressure in a specific case even larger compressed air savings could be achieved, but it would cause a failure to comply with the stroke time requirements. Therefore, all considered combinations meet the set time requirements, although with different quality, and their consideration is not so important for the comparison as the other values. Whereas the capability of the drive to achieve the required pressing force is of the highest importance for the manufacturer, otherwise the task will not be fulfilled. Consequently, the pressing force weight factor has the largest value in this case. The weighting for the remaining two criteria is distributed evenly. 
The value $N_{\text {total }}$ can now be calculated e.g. by the weighted sum of the individual fulfilment degrees $E_{\mathrm{i}}$ or by their multiplication. According to [10], the additive linkage type is more accurate than multiplicative. Hence, the total technical benefit value is defined as follows:

$N_{\text {total }}=\sum_{\mathrm{i}}^{n} w_{\mathrm{i}} \cdot E_{\mathrm{i}}$

\section{TCO analysis}

From the other side, the total cost of ownership should be considered to describe the economic value of a circuit. Following cost parts were calculated:

\section{Table 4: Cost parts of the TCO analysis}

\begin{tabular}{|c|c|}
\hline Cost object & Calculation \\
\hline Acquisition & $\begin{array}{l}\text { based on market list prices of } \\
\text { circuit components; }\end{array}$ \\
\hline Shipping & $\begin{array}{l}\text { based on delivery costs of the } \\
\text { manufacturer and supplier; }\end{array}$ \\
\hline Commissioning & $\begin{array}{l}\text { medium assembly time of one } \\
\text { component ( } 10 \text { min assumed) } \\
\text { multiplied by the component } \\
\text { number and industrial worker } \\
\text { salary; }\end{array}$ \\
\hline Energy costs & $\begin{array}{l}\text { circuit air consumption } \\
\text { multiplied by medium } \\
\text { compressed air price } \\
\left(0,0192 € / \mathrm{Nm}^{3}\right)[11] ;\end{array}$ \\
\hline Maintenance & $\begin{array}{l}\text { assumed as } 5 \% \text { of acquisition } \\
\text { costs; }\end{array}$ \\
\hline Space & $\begin{array}{l}\text { industrial monthly rate for } 1 \mathrm{~m}^{2} \\
\text { space usage multiplied by the } \\
\text { installation area of the drive; }\end{array}$ \\
\hline
\end{tabular}

Dismounting medium disassembly time of one component (5 min assumed) multiplied by the component number and industrial worker salary;

Rate of interest $\quad 10 \%$ assumed [12].

The TCO is accounted for over a period of 5 years.

\section{RESULTS OF THE COST-BENEFIT ANALYSIS}

For each generated combination of the respective task, a lumped-parameter simulation model was created in order to determine the relevant technical properties of a circuit. Subsequently, based on defined utility functions, the total technical benefit of each combination and, according to Table 4, the annual TCO were calculated. The values defined in this way can be graphically represented and compared for each task in the form of a cost-benefit-diagram (see Figures 3 to 6).

Each labelled dot corresponds to a calculated energy saving circuit combination. Combinations that are considered to be optimal for the specific task are highlighted in green and bold, the standard circuits (0) in grey. The optimal variants were chosen as a balance between the achievable technical benefit and the TCO value:

- Task 1: (7b) Filling volumes reduction

- Task 2: (47b) Filling volumes reduction and a pressure-reduced back stroke ( 3 bar $_{\text {abs. }}$ )

- Task 3: (176c) Short circuit with reduced filling volumes and operating pressure (3 bar abs.)

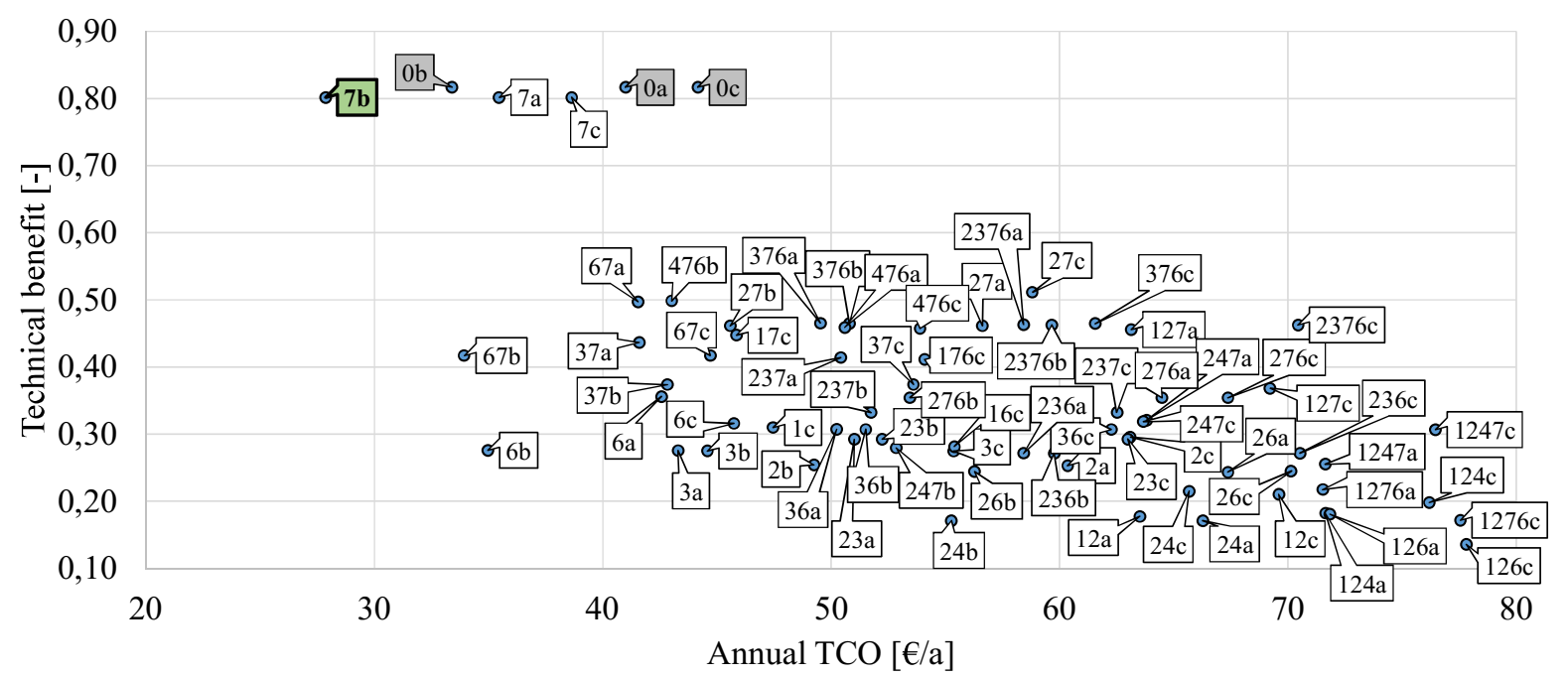

Figure 3: Cost-benefit diagram for the task 1 "Feed drive" 


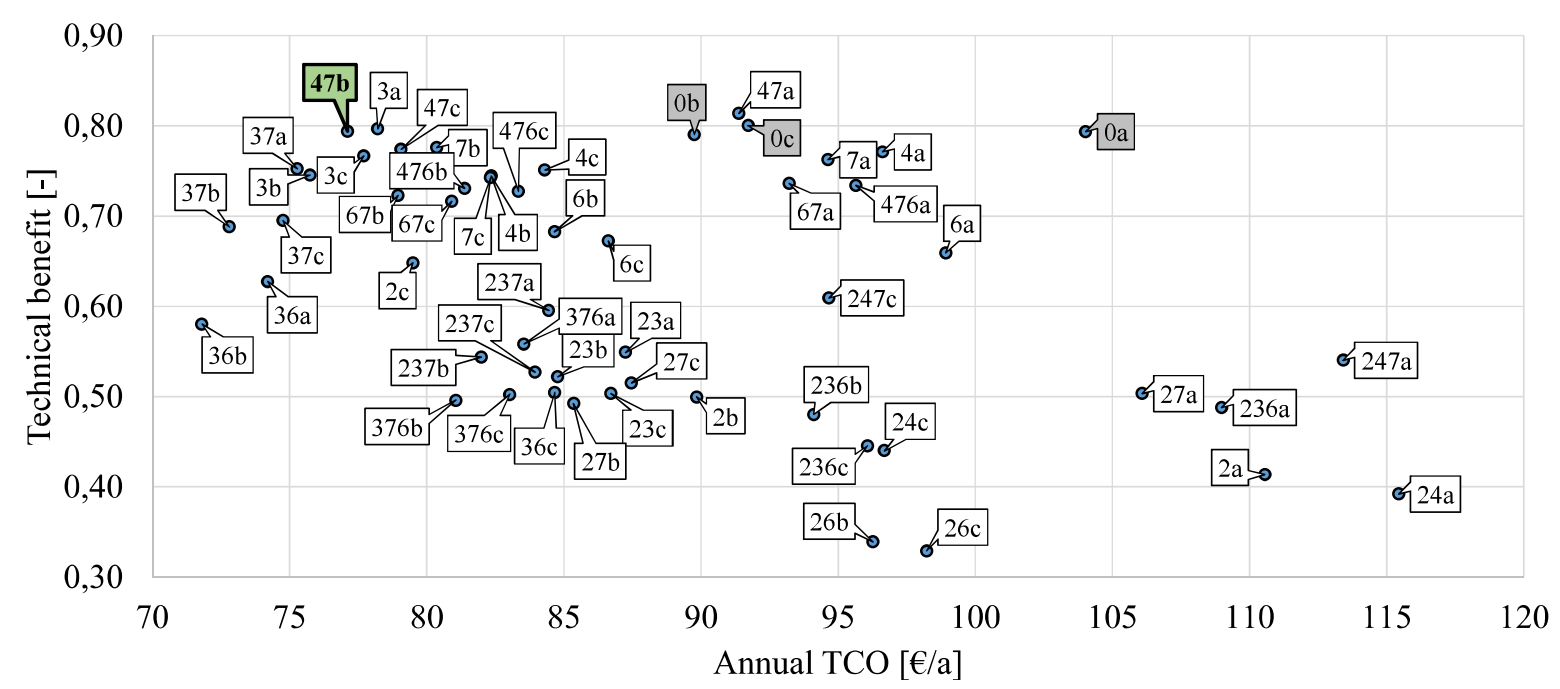

Figure 4: Cost-benefit diagram for the task 2 "Force drive"

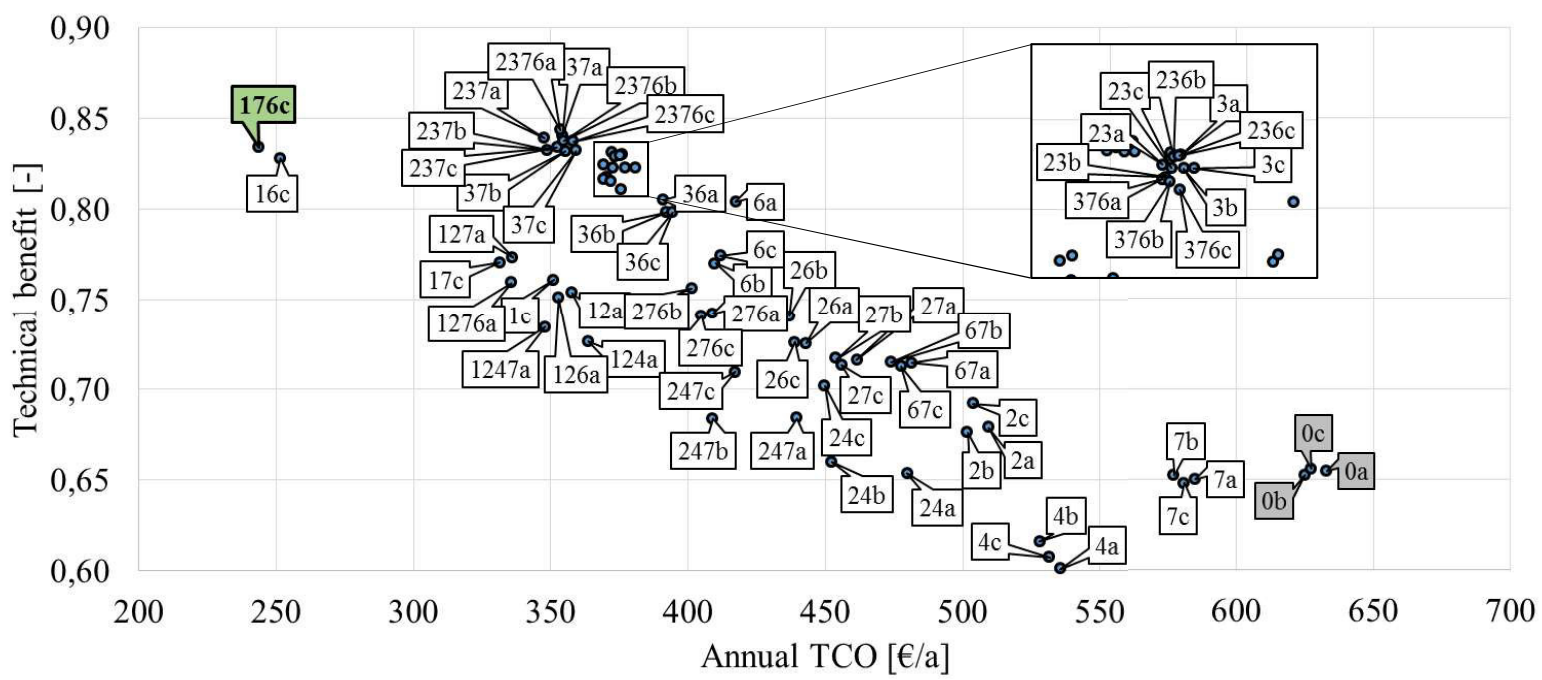

Figure 5: Cost-benefit diagram for the task 3 "Feed drive"

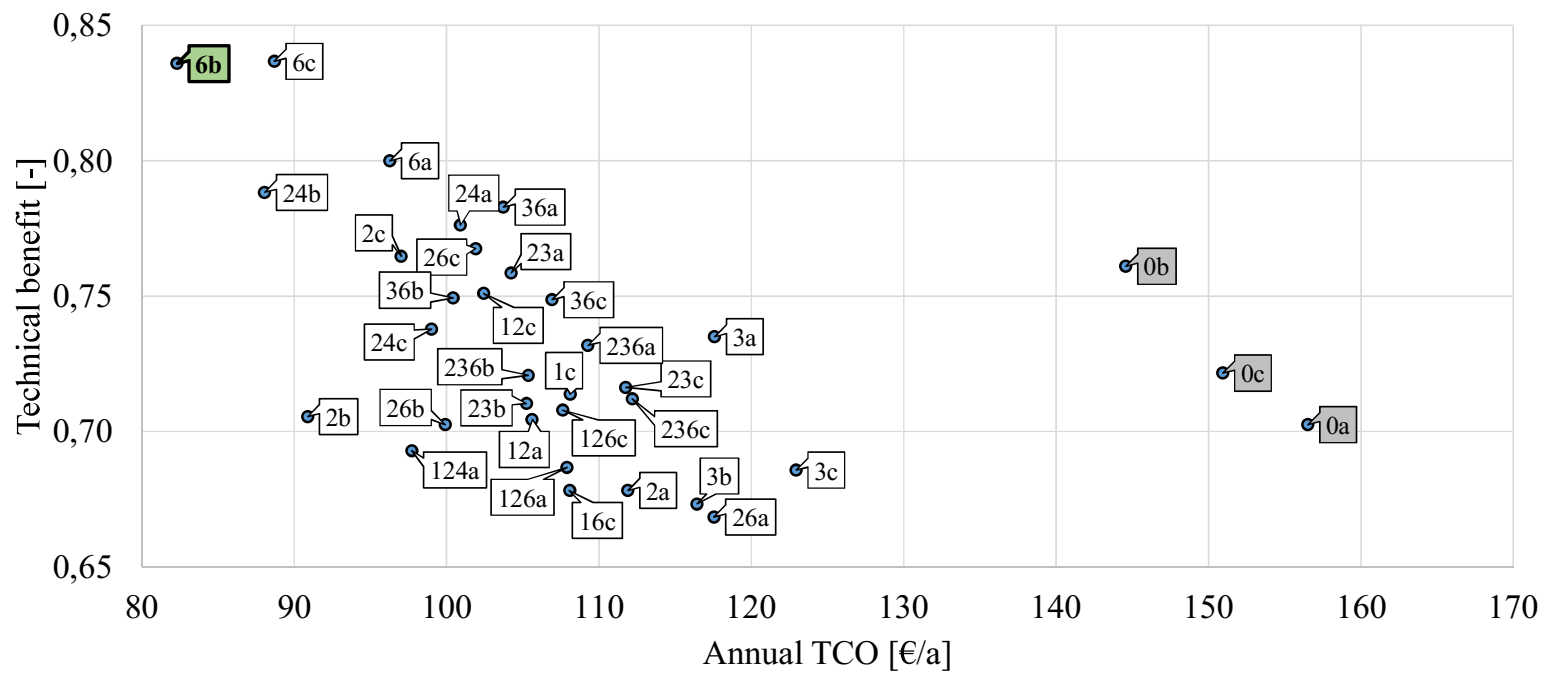

Figure 6: Cost-benefit diagram for the task 4 "Feed drive" 
- Task 4: (6b) General operating pressure reduction ( 2 barabs.)

It can be seen that in the case of smaller and medium-sized handling tasks (up to $25 \mathrm{~mm}$ diameter), large cost savings and an improved technical benefit value can be achieved with simple energy saving measures (reduction of operating pressure (6) and/or filling volumes (7)), which involve relatively few acquisition and assembly costs. In case of the task 2 , even greater TCO savings can be achieved with help of the exhaust air storage (3) and its combinations, however at the expense of the technical value.

For the task 4 too, a simple reduction of the operating pressure was sufficient to increase the technical value and significantly reduce the annual costs. The measure "Reduction of the filling volume" (7) was not considered here, since the tube length was already low in the present task.

The situation is different for the task 3 with a larger-sized cylinder. There, the combination $176 \mathrm{c}$ of a short circuit (1) with other two measures (reduction of filling volumes and operating pressure) has prevailed.

It can be concluded that known energy saving circuits and their combinations are mostly worthwhile for larger cylinders (from a diameter of $32 \mathrm{~mm}$ ) in the considered cases. Although the combination of different savings circuits makes it possible to reach even greater compressed air savings than with individual circuits, the everhigher initial acquisition costs and other cost factors compared to the standard circuit exceed this saving in the most cases thus reducing their technical benefit and even increasing the annual TCO in overall terms.

Especially for the task 1 it can be make clear that although many combinations bring significant savings in compressed air compared to individual circuits, but for their achievement a costly acquisition is necessary, which does not pay in 5 years of observation space (Figure 7).

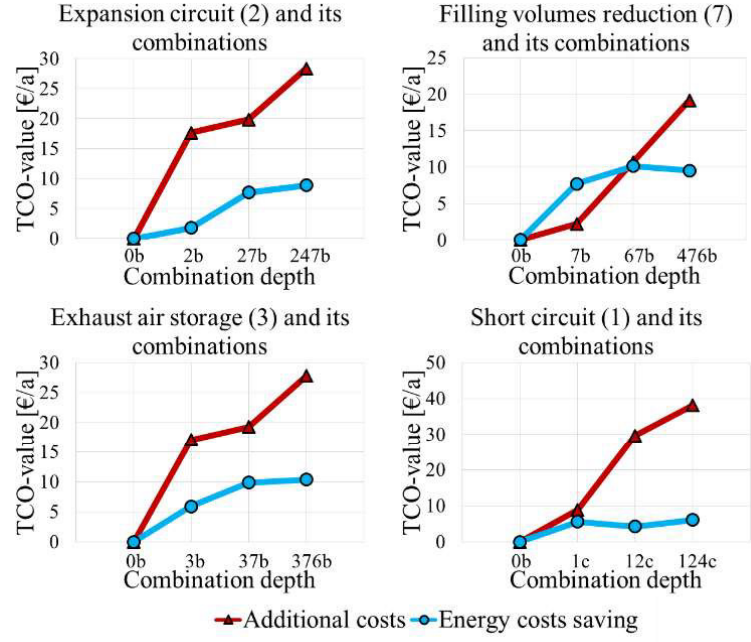

Figure 7: Comparison of energy costs saving and additional costs for each further combination for the task 1

\section{VALIDATION}

In each task, its optimal combination of energy saving measures and the corresponding standard circuit were reconstructed on test benches and measured to validate the calculated mechanical properties and the compressed air saving:

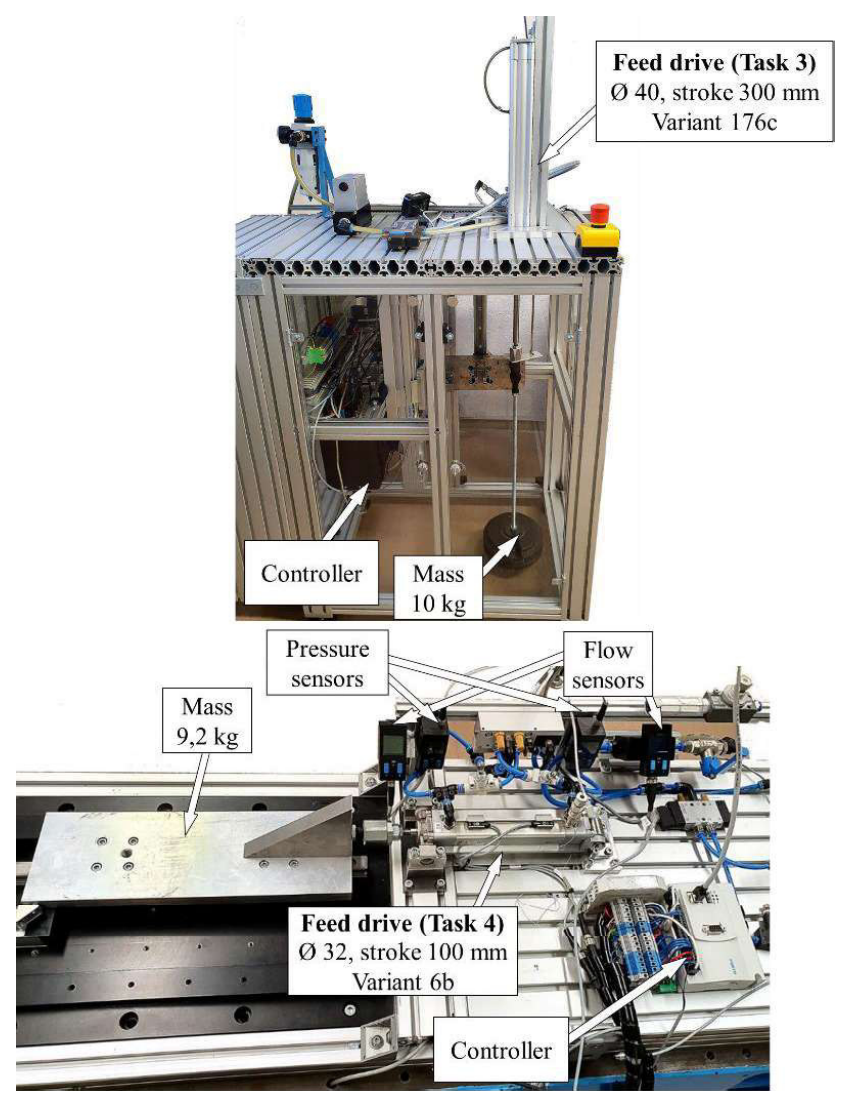

Figure 8: Examples of test benches 
Table 5: Standard and optimal circuits

\begin{tabular}{|c|c|c|c|c|c|c|c|c|}
\hline No. & $\begin{array}{l}\text { Air consur } \\
{[\mathrm{Nl} / \text { cycle }]} \\
\text { simulated }\end{array}$ & $\begin{array}{l}\text { ption } \\
\text { measured }\end{array}$ & $\begin{array}{l}\text { Stroke tim } \\
t_{\text {for }}[\mathrm{s}] \\
\text { simulated }\end{array}$ & $\begin{array}{l}\text { forwards } \\
\text { measured }\end{array}$ & $\begin{array}{l}\text { Stroke tim } \\
t_{\text {back }}[\mathrm{s}] \\
\text { simulated }\end{array}$ & $\begin{array}{l}\text { forwards } \\
\text { measured }\end{array}$ & $\begin{array}{l}\mathrm{TCO} \\
{[€ / \mathrm{a}]}\end{array}$ & $\begin{array}{l}\text { Additional } \\
\text { acquisition } \\
\text { costs }[€]\end{array}$ \\
\hline \multicolumn{9}{|c|}{ 1) Feed drive } \\
\hline $\mathrm{Ob}$ & 0,0801 & 0,089 & 0,015 & 0,014 & 0,010 & 0,010 & 33,40 & 0,00 \\
\hline $7 b$ & 0,0297 & 0,032 & 0,015 & 0,016 & 0,011 & 0,012 & 27,87 & 10,12 \\
\hline \multicolumn{9}{|c|}{ 2) Force drive } \\
\hline $\mathrm{Ob}$ & 0,431 & 0,445 & 0,302 & 0,306 & 0,092 & 0,092 & 89,76 & 0,00 \\
\hline $4 b$ & 0,319 & 0,327 & 0,301 & 0,306 & 0,096 & 0,094 & 82,36 & 30,83 \\
\hline $7 b$ & 0,336 & 0,350 & 0,298 & 0,301 & 0,088 & 0,092 & 80,37 & 20,10 \\
\hline $47 b$ & 0,251 & 0,267 & 0,300 & 0,301 & 0,090 & 0,092 & 77,11 & 50,93 \\
\hline \multicolumn{9}{|c|}{ 3) Feed drive } \\
\hline $0 \mathrm{c}$ & 3,80 & 3,68 & 1,85 & 1,76 & 3,94 & 3,92 & 627,23 & 0,00 \\
\hline $1 \mathrm{c}$ & 1,946 & 1,86 & 1,83 & 1,72 & 3,94 & 3,92 & 351,16 & 30,68 \\
\hline $7 \mathrm{c}$ & 3,47 & 3,27 & 1,84 & 1,71 & 3,90 & 3,87 & 580,95 & 20,10 \\
\hline $6 c$ & 2,23 & 2,14 & 1,87 & 1,77 & 4,01 & 3,96 & 411,86 & 109,02 \\
\hline $17 \mathrm{c}$ & 1,79 & 1,71 & 1,81 & 1,72 & 3,92 & 3,90 & 331,60 & 50,78 \\
\hline $16 \mathrm{c}$ & 1,13 & 1,10 & 1,84 & 1,75 & 4,01 & 3,97 & 251,60 & 139,70 \\
\hline $67 \mathrm{c}$ & 2,63 & 2,54 & 1,86 & 1,78 & 3,98 & 3,93 & 477,70 & 129,12 \\
\hline $176 \mathrm{c}$ & 1,05 & 1,02 & 1,89 & 1,91 & 3,98 & 3,93 & 243,71 & 159,80 \\
\hline \multicolumn{9}{|c|}{ 4) Feed drive } \\
\hline $0 \mathrm{~b}$ & 0,759 & 0,736 & 0,354 & 0,361 & 0,351 & 0,359 & 144,58 & 0,00 \\
\hline $6 \mathrm{~b}$ & 0,298 & 0,315 & 0,349 & 0,353 & 0,340 & 0,344 & 82,34 & 30,83 \\
\hline
\end{tabular}

The results of measurements are shown in Table 5.

As the exergy consumption of a drive cannot be measured directly, the compressed air consumption was measured instead.

The modelling of the end-cushioning system in the cylinder chambers was neglected in this work to reduce the calculation effort. Instead, the simple elastic end stop model (spring-damper element) was assumed. For this reason, the maximum acceleration at the stroke end $a_{\max }$ was determined exclusively simulative.

The pressing force value $F$ at the stroke end for the task 2 was derived from the measured pressure and the cylinder geometry. The force values in this case are:

Table 6: Pressing forces of task 2 combinations

\begin{tabular}{lll}
\hline \multirow{3}{*}{ No. } & \multicolumn{2}{l}{ Pressing force $F$} \\
& Simulation & Measurement \\
\hline $0 \mathrm{~b}$ & 282 & 293 \\
$\mathrm{4b}$ & 279 & 290 \\
$7 \mathrm{~b}$ & 281 & 294 \\
$47 \mathrm{~b}$ & 278 & 292 \\
\hline
\end{tabular}

The TCO balance cannot be validated because of its long-term observation.

As the measured and calculated values agree well, the calculated savings could be proven. The deviations of the air consumption are due to the problematic consideration of dead volumes of a real system and inaccuracy of the flow sensors. In particular, the dead volumes of the cylinder and the pressure sensors for the simulation were estimated from their geometry and might not completely correspond to real values.

\section{CONCLUSION AND OUTLOOK}

In the present article, a range of combinations of energy saving measures and circuits in the pneumatics has been studied using the example of four common automation tasks. Cumulative effects of combining could be demonstrated: in the vast majority of cases, an additional measure increases the compressed air savings. With each further combination, higher savings can be achieved than with the individual circuits. However, the overall balance shows that these savings are often offset by the additional costs and/or deteriorated mechanical properties. Nevertheless, there could be also found combinations that make it not only possible to reduce the TCO drastically, but at least sustain or even improve the mechanical properties in general.

The research results can be transferred to similar tasks in pneumatics. The presented comprehensive approach of the task analysis can serve as example of how an optimal configuration for the specific task can be chosen thus creating a 
basis for solving constructive challenges in designing of pneumatic automation systems.

It could also be possible to enhance the presented combinatorial approach e.g. by means of a needs-oriented parameter optimisation (determining its optimum cylinder piston diameter etc.) The article aimed, though, to make recommendations primarily for already given fixed system parameters such as cylinder geometry.

\section{NOMENCLATURE}

$a_{\max } \quad$ Maximum acceleration at the stroke end $\left[\mathrm{m} / \mathrm{s}^{2}\right]$

$c_{\mathrm{p}} \quad$ Specific heat capacity at constant pressure $[\mathrm{J} /(\mathrm{kg} \cdot \mathrm{K})]$

E Fulfilment degree [-]

Ex Exergy consumption [J/cycle]

$F \quad$ Pressing force [N]

$l \quad$ Hose length [m]

$m \quad$ Load mass [kg]

$\dot{m}_{1} \quad$ Mass flow at the circuit inlet $[\mathrm{kg} / \mathrm{s}]$

$N_{\text {total }} \quad$ Total technical benefit value [-]

$p \quad$ Pressure $[\mathrm{Pa}]$

$p_{0} \quad$ Atmospheric pressure $[\mathrm{Pa}]$

$p_{1} \quad$ Air pressure at the circuit inlet $[\mathrm{Pa}]$

$R_{s} \quad$ Specific gas constant $[\mathrm{J} /(\mathrm{kg} \cdot \mathrm{K})]$

$S_{\text {force }} \quad$ Pressing force safety factor at the stroke end [-]

$t_{\text {back }} \quad$ Stroke time backwards [s]

$t_{\text {for }} \quad$ Stroke time forwards [s]

$t_{\mathrm{p}} \quad$ Payback time [a]

$T_{0} \quad$ Ambient temperature $[\mathrm{K}]$

$T_{1} \quad$ Air temperature at the circuit inlet $[\mathrm{K}]$

TCO Total cost of ownership

$w \quad$ Weight factor [\%]

\section{REFERENCES}

[1] Barroso JM (2010) Communication from the commission - Europe 2020 - A strategy for smart, sustainable and inclusive growth, Brussels, 2010
[2] NN (2015) Schunk Mikroventile. Installation and operating instructions, SCHUNK GmbH \& Co. KG, Lauffen/Neckar

[3] Gauchel W (2006) Energiesparende Pneumatik. O+P Ölhydraulik \& Pneumatik, 1/2006:33-39

[4] Hepke J, Weber J (2013) Energy saving measures on pneumatic drive systems. Paper presented at the 13th Scandinavian International Conference on Fluid Power, SICFP'13, Linköping, Sweden, 4-9 June 2013

[5] Doll M, Sawodny O, Neumann R (2012) Energy efficient adaptive control of pneumatic drives with switching valves. Paper presented at the 8 th International Fluid Power Conference, SICFP'13, Dresden, Germany, 26-28 March 2012

[6] Kawakami Y, Terashima Y, Kawai S (1999) Application of energy-saving to pneumatic driving systems. Paper presented at the 4th JHPS international symposium on fluid power, Tokio, Japan, 15-17 November 1999

[7] NN (2012) EnEffAH - Energy efficiency in production in the drive and handling technology field. Funding code 0327484A, final report, Esslingen, Germany

[8] Hepke J (2017) Energetische Untersuchung und Verbesserung der Antriebstechnik pneumatischer Handhabungssysteme. Dissertation, Technical University of Dresden

[9] Arinaga T et al. (2000). Approach for energysaving of pneumatic systems. Proceedings of $1 \mathrm{st}$ FPNI-PhD Symposium Hamburg 2000, p. 49-56

[10] Rinza P, Schmitz H (1992) Nutzwert-KostenAnalyse: eine Entscheidungshilfe. VDI-Verlag, Düsseldorf

[11] NN (2014) Energiekosten in Druckluftsystemen bis zu $60 \%$ senken. White Paper Festo, Festo AG \& Co. KG, Esslingen

[12] Wöltje J (2011) Betriebswirtschaftliche Formelsammlung. Haufe-Lexware, Freiburg 